\title{
Challenges surrounding postoperative adjuvant chemotherapy for T2N0 gastric cancer (Review)
}

\author{
KE-KANG SUN ${ }^{1,2^{*}}$, QING-HUA WANG ${ }^{1 *}$ and YONG-YOU WU ${ }^{2}$ \\ ${ }^{1}$ Department of Gastrointestinal Surgery, Affiliated Kunshan Hospital to Jiangsu University, Suzhou, Jiangsu 215300; \\ ${ }^{2}$ Department of Gastrointestinal Surgery, The Second Affiliated Hospital of \\ Soochow University, Suzhou, Jiangsu 215008, P.R. China
}

Received April 8, 2020; Accepted July 23, 2020

DOI: $10.3892 / \mathrm{ol} .2020 .11985$

\begin{abstract}
Determining the requirement for adjuvant chemotherapy in patients with stage IB gastric cancer (GC), and particularly for those with stage T2N0 (muscularis propria) disease, remains challenging. Patients with stage II/III disease benefit from postoperative adjuvant therapy; however, the randomized trials examining whether such therapy affords any survival benefit to patients with T2N0 disease are not sufficient. Current evidence suggests that not all patients with T2N0 disease should undergo such treatment, but only those with a high risk. To date, a number of retrospective studies have attempted to identify factors that are predictive of increased risk in an effort to guide adjuvant therapy-related clinical decision making. The National Comprehensive Cancer Network and the Chinese Society of Clinical Oncology have published guidelines regarding factors associated with increased patient risk. As a result, treatment decisions for patients with stage T2N0 disease are currently determined on an individualized basis, in light of risk factors and the potential benefits
\end{abstract}

Correspondence to: Professor Yong-You Wu, Department of Gastrointestinal Surgery, The Second Affiliated Hospital of Soochow University, 1,055 Sanxiang Road, Suzhou, Jiangsu 215008, P.R. China

E-mail:wuyoyo@aliyun.com

*Contributed equally

Abbreviations: GC, gastric cancer; DFS, disease-free survival; RFS, relapse-free survival; OS, overall survival; AJCC, American Joint Committee on Cancer; JGCG, Japanese gastric cancer treatment guidelines; ESMO, European Society of Medical Oncology; NCCN, National Comprehensive Cancer Network; CSCO, Chinese Society of Clinical Oncology; ARTIST, Adjuvant Chemoradiation Therapy in Stomach Cancer; ACTS-GC, Adjuvant Chemotherapy Trial of TS-1 for Gastric Cancer; CLASSIC, Capecitabine and Oxaliplatin Adjuvant Study in Stomach Cancer; HER, Human Epidermal Growth Factor Receptor

Key words: gastric cancer, adjuvant chemotherapy, T2, muscularis propria, prognosis of treatment. The present review surveyed current evidence related to the treatment of patients with high-risk GC and highlighted the potential avenues for future investigated.

\section{Contents}

1. Introduction

2. Unnecessary adjuvant chemoradiotherapy for the treatment of T2N0 GC

3. Postoperative adjuvant chemotherapy for stage II/III GC

4. Controversy regarding adjuvant chemotherapy in patients with T2N0 GC

5. Predictive markers for adjuvant chemotherapy in T2N0 GC

6. Other markers for adjuvant chemotherapy in T2N0 GC

7. Conclusion

\section{Introduction}

Patients with gastric cancer (GC) and patients with locally advanced and node-positive disease can benefit from adjuvant therapy following resection (1). At present, adjuvant treatment recommendations are made in light of risk stratification guidelines based upon the American Joint Committee on Cancer (AJCC) staging classification system (2). The eighth revision of the AJCC staging system in 2017 reclassified tumors that invade the subserosa as T3 disease, whereas these tumors were previously classified as stage T2b disease (2). To date, the majority of randomized trials conducted to evaluate the value of adjuvant therapy in patients with GC have been focused on patients with stage II/III disease $(3,4)$. To the best of our knowledge, no randomized trials have specifically assessed whether adjuvant therapy offers survival benefits to those with stage T2N0 disease. Thus, the appropriate adjuvant therapy of choice for the treatment of these patients remains a matter of controversy. The Japanese gastric cancer treatment guidelines (JGCG) do not recommend adjuvant therapy for those with stage IB (T1N1 and T2N0) GC (5), whereas the European Society of Medical Oncology (ESMO) guidelines recommend postoperative adjuvant chemotherapy or chemoradiotherapy for those with stage IB GC (6). The National 
Comprehensive Cancer Network (NCCN) (7) and the Chinese Society of Clinical Oncology (CSCO) (8) have also provided recommendations regarding the administration of adjuvant chemotherapy only to patients exhibiting specific findings associated with high-risk disease, such as younger age ( $\leq 50$ or $\leq 40$ years, respectively), poorly differentiated/high grade cancer, lymphovascular invasion, neural invasion or $<\mathrm{D} 2$ lymph node dissection. These inconsistencies have been further complicated by nation-to-nation differences in lymph node dissection strategies. Currently, adjuvant therapy is not thought to be appropriate for all patients with stage T2N0 GC following radical tumor resection; instead, this treatment is only administered to those with high-risk disease (9). In the present review, the features of stage T2N0 disease that may assist with the identification of patients who are most likely to benefit from the application of adjuvant chemotherapy were discussed.

\section{Unnecessary adjuvant chemoradiotherapy for the treatment of T2No GC}

In the North American Intergroup-0116 trial, a total of 556 patients with resected adenocarcinoma of the stomach or gastroesophageal junction were evaluated as a single cohort of individuals with stage IB or higher resectable GC. In the trial, a 10-year follow-up confirmed that postoperative chemoradiotherapy was associated with sustained and robust benefits in overall survival time $(9,10)$. As a result, adjuvant chemoradiotherapy is the standard regimen used to treat patients with GC, who have undergone curative surgical resection and have stage $\mathrm{T} 3$ or higher disease and/or positive nodal disease in the USA. The patients classified with stage T2a/bN0 disease according to the sixth edition AJCC staging criteria (11) were reclassified with stage T2N0 (stage IB) or stage T3N0 (stage IIA) disease using the 8th edition revision of this staging system (2). A retrospective examination suggested that very few patients who met the revised criteria for stage T2N0 GC were included in the North American Intergroup-0116 trial, and these results cannot be interpreted as demonstrating the benefits of chemotherapy in patients with T2N0 disease. Only $10 \%$ of patients had undergone formal D2 dissection in the trial, while $90 \%$ had undergone D0 or D1 lymph node dissection (10). Therefore, the trial lacked the power to reliably determine whether postoperative chemoradiotherapy was beneficial to patients with D2 lymph node dissection.

The adjuvant chemoradiation therapy in stomach cancer (ARTIST) trial analyzed 458 patients with GC, higher than stage IB (excluding those with T2aN0 disease) who had undergone D2 lymph node dissection (12). Patients in this trial were randomized into chemotherapy and chemoradiotherapy groups. No differences in disease-free survival (DFS) or overall survival (OS) times were detected between these two groups upon 3 and 7-year follow-up $(12,13)$. In light of these results, the ESMO guidelines suggested that postoperative chemoradiotherapy was not necessary for patients with stage IB or higher GC following appropriate surgical resection, including D2 lymph node dissection (6). A subgroup-based analysis of the trial results suggested that adjuvant chemoradiotherapy similarly afforded no notable benefits relative to adjuvant chemotherapy alone in patients with stage IB disease (14). This analysis did not include patients with stage T2N0 (muscularis propria) disease; therefore, we hypothesized that these patients would similarly not benefit from postoperative radiotherapy, as this stage of the disease was even earlier compared with those included in the ARTIST trial. A retrospective analysis also suggested that patients with stage III/IV GC would benefit from radiotherapy, whereas patients with stage IB and II tumors would not (15). Therefore, chemoradiotherapy would not be required for all patients with T2N0 GC following D2 gastrectomy.

\section{Postoperative adjuvant chemotherapy for stage II/III GC}

The adjuvant chemotherapy trial of TS-1 for gastric cancer (ACTS-GC) incorporated 1,059 patients who were randomly assigned to either undergo surgery only or with S-1 adjuvant chemotherapy (3). The patients included in the study had histologically-confirmed stage II (excluding T1), IIIA, or IIIB GC (defined based on the 13th edition of JCGC criteria) (16) and had undergone R0 gastrectomy with extended D2 lymphadenectomy. Postoperative S-1 adjuvant chemotherapy could improve OS and relapse-free survival (RFS) times in individuals with stage II/III GC who had undergone D2 gastrectomy $(3,17)$. This phase III trial was the first to conclusively demonstrate that adjuvant chemotherapy was an effective means of treating GC, following D2 gastrectomy. The capecitabine and oxaliplatin adjuvant study in stomach cancer (CLASSIC) trial was designed in an effort to assess the DFS benefits of capecitabine and oxaliplatin-based adjuvant chemotherapy following D2 gastrectomy in patients with GC (4). This trial enrolled patients with histologically-confirmed stage II (T2N1, T1N2 and T3N0), IIIA (T3N1, T2N2 and T4N0), or IIIB (T3N2) gastric adenocarcinoma (based on the 6th edition AJCC staging system). The results of the CLASSIC trial demonstrated that postoperative capecitabine and oxaliplatin treatment improved OS and DFS times compared with that in surgery alone in patients with advanced GC $(4,18)$.

The Global Advanced/Adjuvant Stomach Tumor Research International Collaboration group performed a meta-analysis of the findings from 17 trials based on individual patient data and determined that adjuvant chemotherapy was associated with significant improvements in OS [hazard ratio (HR), 0.82; $\mathrm{P}<0.001]$ and DFS times (HR, 0.82; $\mathrm{P}<0.001)$ compared with that in surgery alone (19). However, the vast majority of the data in the study was focused on patients with stage II/III disease. Therefore, there were too few patients with T2NO GC included in the trials to conduct a conclusive assessment of the relative benefits of adjuvant chemotherapy in these patients. The CLASSIC and ACTS-GC trials did not include patients with stage T2N0 $\mathrm{GC}$, according to 8th edition of the AJCC criteria. At present, to the best of our knowledge, no randomized prospective clinical trials have specifically evaluated the relative benefits of adjuvant chemotherapy in patients with stage T2N0 GC.

\section{Controversy regarding adjuvant chemotherapy in patients with T2NO GC}

A retrospective analysis of 2,229 patients with (stage I-IV) in the 2001-2008 California Cancer Registry determined 
that $29 \%$ of these patients exhibited N0 disease, while $70 \%$ exhibited T1-T2 tumors (20). Multivariate analyses indicated that surgery alone was associated with improved survival outcomes compared with that in adjuvant therapy in this study cohort; therefore, adjuvant therapy was not beneficial to patients with stage IB disease. A separate evaluation of the Surveillance Epidemiology of End Results (SEER) database similarly examined adjuvant therapy-related outcomes in patients with stage IB GC from 1988 to 2008 (21). The analysis suggested that survival rates were highest among patients with stage IB GC, who had received chemoradiotherapy compared with that in patients receiving surgery alone, when patients with stage IA disease were omitted. Therefore, these results supported adjuvant therapy administration to patients with stage IB GC (21). Another retrospective assessment of 23,461 patients with stage IB-II GC in the National Cancer Database (1998-2011) revealed that the risk-adjusted mortality rates among patients with stage IB GC were notably higher when patients did not undergo adjuvant therapy, although no OS benefit to adjuvant chemoradiotherapy was detected when adequate lymph node dissection was conducted in patients with node-negative disease (22). These findings suggested that the benefit of adjuvant therapy for patients with stage T2N0 $\mathrm{GC}$, following adequate lymphadenectomy remains uncertain. These retrospective studies also have numerous limitations, including the fact that they included patients that would now be classified with T1N1 or T3N0 stage disease based on the current AJCC staging system (2). Further randomized trials would be important to adequately evaluate the benefits of adjuvant therapy in patients with stage T2N0 GC. These patients typically have a favorable prognosis; however, several retrospective studies suggested that specific subgroups of high-risk patients with T2N0 GC had poorer prognosis (7,8,23-25). Therefore, prognostic markers should be identified to assist with identifying which patients with stage T2N0 GC would benefit from adjuvant chemotherapy.

\section{Predictive markers for adjuvant chemotherapy in T2N0 GC}

According to the ESMO guidelines, postoperative adjuvant chemotherapy is recommended in patients with T2N0 disease, that have undergone surgery without administration of preoperative chemotherapy (6). However, under the JGCG guidelines, these patients are recommended to only undergo observation without adjuvant treatment following curative surgical resection (5). In addition, the NCCN and CSCO have published guidelines describing these high-risk characteristics; adjuvant chemotherapy may reduce the risk of metastasis in the following groups of patients with T2N0: Younger age ( $\leq 40$ or $\leq 50)$, poorly differentiated/higher grade cancer, lymphovascular invasion, neural invasion or $<$ D2 lymph node dissection $(3,4)$.

Lymphovascular and/or perineural invasion. The invasion of the lymphovascular system was associated with metastatic tumor progression, whereas perineural invasion represents another route of tumor spread (26). Stratified analyses based on tumor stage have not been conducted in patients with GC exhibiting such invasion; however, its presence would be predictive of poorer patient survival and could be used to guide planning on adjuvant chemotherapy treatment. A prospective study of 233 patients with stage T2N0 GC, who had undergone D2 lymph node dissection, found that the 5-year survival rates were lower in patients exhibiting lymphovascular or perineural invasion (hazard ratio (HR), 3.09; $\mathrm{P}=0.025$ and $\mathrm{HR}$, 4.83; $\mathrm{P}=0.009$, respectively) compared with patients without lymphovascular or perineural invasion (24). A separate retrospective assessment of 225 patients with GC, who had received $\mathrm{R} 0$ resection, determined that lymphovascular $(\mathrm{P}=0.001)$ and perineural $(\mathrm{P}<0.001)$ invasion were independent predictors of reduced OS time (23). In addition, the study also found that adjuvant chemotherapy was associated with significant improvements in the OS time of patients with these invasive phenotypes $(\mathrm{P}<0.001)(23)$. Consistent with these results, Araki et al (27) found that in a retrospective assessment of 130 patients with stage IB node-negative (T2N0) GC venous invasion was independently predictive of reduced patient RFS and $\mathrm{OS}$ times (HR, 3.00; $\mathrm{P}=0.035$ and $\mathrm{HR}, 5.00 ; \mathrm{P}=0.006$, respectively).

Poorly differentiated/higher-grade cancer. A retrospective analysis of 2,783 patients with stage I GC, who had undergone surgery, revealed that poorer tumor differentiation was associated with reduced RFS $(\mathrm{P}=0.001)(25)$. In another retrospective assessment of 86 patients with stage IB GC, Yokoyama et al (28) determined that a histologically undifferentiated-type of adenocarcinoma $(\mathrm{P}=0.0069)$ was independently associated with the risk of tumor recurrence. Future randomized controlled studies would be essential to validate these findings; however, patients with stage IB GC are likely to exhibit poorer tumor differentiation and would benefit from adjuvant chemotherapy.

Inadequate lymphadenectomy. Inadequate lymphadenectomy was independently predictive of poorer long-term outcomes in patients with GC, even in those who received adjuvant therapy (29). Retrospective analysis of the National Cancer Database determined that surgery was only associated with poorer OS times in patients with inadequately staged stage IB $\mathrm{GC}(\mathrm{HR}, 1.24 ; \mathrm{P}=0.003)$, whereas the same was not true in those with appropriately staged disease (22). A SEER population-based study found that the examination of 15 or fewer lymph nodes in patients with stage IB GC was independently predictive of poorer OS time (30). In line with these findings, another retrospective evaluation of 1,687 patients with stage T2N0 GC revealed that the 5-year OS time in patients whom 15 or more lymph nodes had been examined was 71 vs. $53 \%$ in patients who had $<15$ nodes examined $(\mathrm{P}<0.001)$ (31). Furthermore, adjuvant chemoradiotherapy improved the OS time of patients who had $<15$ nodes evaluated ( $\mathrm{HR}, 0.71 ; \mathrm{P}=0.043$ ), whereas no such benefit was observed in patients whom $\geq 15$ nodes had been evaluated (31). These results emphasize the value of considering inadequate lymphadenectomy, as another potential marker of higher-risk disease and could potentially explain the benefits of adjuvant chemotherapy in at least some patients with stage T2N0 disease.

\section{Other markers for adjuvant chemotherapy in T2N0 GC}

Tumor location. A prospective analysis of 103 patients with stage IB GC revealed that tumor location was the only 
substantial factor associated with patient prognosis using univariate and multivariate analyses (32). Tumors in the upper third of the stomach were associated with an $81.8 \%$ 5-year OS time, whereas tumors in the middle or low third of the stomach were associated with a 95.5\% 5-year OS time $(\mathrm{P}=0.0093)$. A separate retrospective analysis identified a high-risk subgroup among 225 patients with stage T2N0 GC, who would benefit from adjuvant chemotherapy (23). The study observed that tumor location within the upper third of the stomach was independently associated with a decrease in the OS time of these patients $(\mathrm{P}<0.001)$, and the patients were also reported to benefit from adjuvant chemotherapy treatment (23).

Microsatellite instability (MSI). GC is a highly heterogenous disease as evidenced by the GC molecular subclassification data in The Cancer Genome Atlas (33). Patients with GC exhibiting MSI subtype disease had an improved OS time compared with patients without such genomic instability (34). A systematic meta-analysis of 48 studies enrolling 18,612 patients with GC observed that patients with MSI-high GC had a significantly improved OS time compared with that in patients with microsatellite stability (HR, 0.69; $\mathrm{P}<0.001)$ (30). Choi et al (35) further evaluated MSI and programmed cell death 1 protein and mRNA expression in 592 patients in the CLASSIC trial and revealed that MSI-high status was independently associated with patient DFS time using multivariate analysis (HR, 0.301; $\mathrm{P}=0.008$ ). Adjuvant chemotherapy was associated with enhanced DFS time in patients exhibiting microsatellite stability, but it was not associated with any benefit in patients with high MSI (35). The post hoc analyses from the ARTIST trial in Asian patients also found that MSI-high status was associated with improved survival outcomes in patients with GC compared with MSI-low status (36). However, a separate retrospective analysis of 429 patients reported that adjuvant chemoradiotherapy was associated with notably improvements in the OS times of patients with stage III GC, respective of MSI status (37). By contrast, only patients with stage IB/II GC, exhibiting microsatellite stability benefited from chemoradiotherapy, whereas no benefit was observed in MSI-high patients with stage IB/II disease. These findings suggested that postoperative chemotherapy may not be warranted in patients with stage T2N0 GC, exhibiting MSI-high disease (38).

Gene expression profiling. Gene expression profiling has been utilized to evaluate the prognosis of patients with breast cancer and predict patient treatment responses (39). However, gene expression profiling has not been used for the clinical evaluation of patients with GC. A retrospective analysis compared the gene expression profiles of patients with stage II/III GC, who had undergone S-1 adjuvant chemotherapy, following gastrectomy and identified 147 upregulated and 192 downregulated genes associated with favorable OS and DFS outcomes (40). However, the same genes were not associated with the aforementioned outcomes in patients who did not undergo S-1 adjuvant chemotherapy; therefore, these genes may represent biomarkers, that could predict which patients with stage II/III GC would benefit from adjuvant chemotherapy (40). A separate retrospective analysis of patients in the CLASSIC trial identified four classifier genes (granzyme B, tryptophanyl-tRNA synthetase 1, caudal-type homeobox 1 and secreted frizzled-related protein 4), which could be used to predict whether or not patients would benefit from chemotherapy (41). The patients who were predicted to benefit from chemotherapy, based on this four-gene signature and underwent adjuvant chemotherapy exhibited significantly improved 5-year OS times compared with those that only underwent surgery alone $(\mathrm{P}=0.0015)$. However, no comparable improvements were observed in patients who were not predicted to benefit from chemotherapy.

Human epidermal growth factor receptor (HER). HER and HER ligand protein and mRNA expression were detected in breast cancer, and the increased expression of these factors was associated with a more aggressive disease (42). A retrospective tissue microarray analysis of 221 patients with GC demonstrated that HER 2 and HER3 amplification was significantly associated with poorer OS times compared with that in patients without any amplification $(\mathrm{P}=0.023)(43)$. Consistent with these results, a meta-analysis of 19 studies, that incorporated 4,342 patients with GC patients observed that HER2+ patients with GC had worse OS and DFS outcomes in 15 studies, using a univariate analysis (HR, 1.59), and this finding was also found in seven studies, in a further multivariate analysis (HR, 1.58), although the results were not statistically significant (44). Therefore, increased HER expression is associated with the prognosis of patients with advanced GC. However, further research would be required to evaluate the association among dysregulation, survival outcomes, and appropriate treatment strategies in patients with T2NO GC.

\section{Conclusion}

At present, no prospective randomized trials have firmly established whether adjuvant chemotherapy provides any survival benefit to patients with stage T2N0 GC. Typically, the addition of radiotherapy, as an adjuvant chemotherapy regimen is unnecessary in such patients following D2 gastrectomy. Retrospective analyses have found that adjuvant chemotherapy would not be appropriate for all patients with T2NO GC and should instead be restricted to high-risk patients (23-25). However, these prior trials were sub-optimally designed. At the Affiliated Kunshan Hospital to Jiangsu University, evidence-based guidelines established in light of the NCCN and CSCO recommendations would be followed and patients would be encouraged to participate in clinical trials as appropriate. Future guidelines have the potential to refine current clinical guidance for the treatment of patients with stage T2N0 GC by incorporating additional markers and prospective studies.

\section{Acknowledgements}

Not applicable.

\section{Funding}

The present study was supported by the Gastrointestinal oncology international team cooperation project (grant no. SZYJTD201804).

\section{Availability of data and materials}

Not applicable. 


\section{Authors' contributions}

KS contributed to literature search and the writing of the manuscript. QW participated in the study design and literature search. YW contributed to the study design. All authors read and approved the final manuscript.

\section{Ethics approval and consent to participate}

Not applicable.

\section{Patient consent for publication}

Not applicable.

\section{Competing interests}

The authors declare that they have no competing interests.

\section{References}

1. Macdonald JS, Smalley SR, Benedetti J, Hundahl SA, Estes NC Stemmermann GN, Haller DG, Ajani JA, Gunderson LL, Jessup JM and Martenson JA: Chemoradiotherapy after surgery compared with surgery alone for adenocarcinoma of the stomach or gastroesophageal junction. N Engl J Med 345: 725-730, 2001.

2. Seeruttun SR, Yuan S, Qiu H, Huang Y, Li Y, Liang Y, Guan Y, Zhan Y,Li W, Chen Y, et al: A comprehensive analysis comparing the eighth AJCC gastric cancer pathological classification to the seventh, sixth, and fifth editions. Cancer Med 6: 2804-2813, 2017

3. Sakuramoto S, Sasako M, Yamaguchi T, Kinoshita T, Fujii M, Nashimoto A, Furukawa H, Nakajima T, Ohashi Y, Imamura $\mathrm{H}$, et al: Adjuvant chemotherapy for gastric cancer with S-1, an oral fluoropyrimidine. N Engl J Med 357: 1810-1820, 2007.

4. Bang YJ, Kim YW, Yang HK, Chung HC, Park YK, Lee KH Lee KW, Kim YH, Noh SI, Cho JY, et al: Adjuvant capecitabine and oxaliplatin for gastric cancer after D2 gastrectomy (CLASSIC): A phase 3 open-label, randomised controlled trial Lancet 379: 315-321, 2012.

5. Japanese Gastric Cancer Association: Japanese gastric cancer treatment guidelines 2014 (ver. 4). Gastric Cancer 20: 1-19, 2017.

6. Smyth EC, Verheij M, Allum W, Cunningham D, Cervantes A and Arnold D; ESMO Guidelines Committee: Gastric cancer: ESMO clinical practice guidelines for diagnosis, treatment and follow-up. Ann Oncol 27 (Suppl 5): v38S-v49S, 2016.

7. Ajani JA, D'Amico TA, Baggstrom M, et al: Gastric cancer, version 5.2017. NCCN Clinical Practice Guidelines in Oncology 14: 1286-1312, 2017.

8. Wang FH, Shen L, Li J, Zhou ZW, Liang H, Zhang XT, Tang L, Xin Y, Jin J, Zhang YJ, et al: The chinese society of clinical oncology (CSCO): Clinical guidelines for the diagnosis and treatment of gastric cancer. Cancer Commun (Lond) 39: 10, 2019

9. Smalley SR, Benedetti JK, Haller DG, Hundahl SA, Estes NC, Ajani JA, Gunderson LL, Goldman B, Martenson JA, Jessup JM, et al: Updated analysis of SWOG-directed intergroup study 0116: A phase III trial of adjuvant radiochemotherapy versus observation after curative gastric cancer resection. J Clin Oncol 30: 2327-2333, 2012

10. Hundahl SA, Macdonald JS, Benedetti J and Fitzsimmons T; Southwest Oncology Group and the Gastric Intergroup: Surgical treatment variation in a prospective, randomized trial of chemoradiotherapy in gastric cancer: The effect of undertreatment. Ann Surg Oncol 9: 278-286, 2002.

11. Choi KH, Kim BS, Oh ST, Yook JH and Kim BS: Comparison the sixth and seventh editions of the AJCC staging system for T1 gastric cancer: A long-term follow-up study of 2124 patients. Gastric Cancer 20: 43-48, 2017.

12. Lee J, Lim DH, Kim S, Park SH, Park JO, Park YS, Lim HY, Choi MG, Sohn TS, Noh JH, et al: Phase III trial comparing capecitabine plus cisplatin versus capecitabine plus cisplatin with concurrent capecitabine radiotherapy in completely resected gastric cancer with D2 lymph node dissection: The ARTIST trial. J Clin Oncol 30: 268-273, 2012.
13. Park SH, Sohn TS, Lee J, Lim DH, Hong ME, Kim KM, Sohn I, Jung SH, Choi MG, Lee JH, et al: Phase III trial to compare adjuvant chemotherapy with capecitabine and cisplatin versus concurrent chemoradiotherapy in gastric cancer: Final report of the adjuvant chemoradiotherapy in stomach tumors trial, including survival and subset analyses. J Clin Oncol 33: 3130-3136, 2015.

14. Kim Y, Kim KM, Choi MG, Lee JH, Sohn TS, Bae JM, Kim S, Lee SJ, Kim ST, Lee J, et al: Adjuvant chemotherapy with or without concurrent radiotherapy for patients with stage IB gastric cancer: A subgroup analysis of the adjuvant chemoradiotherapy in stomach tumors (ARTIST) phase III trial. J Gastric Cancer 18: 348-355, 2018.

15. Coburn NG, Govindarajan A, Law CH, Guller U, Kiss A, Ringash J, Swallow CJ and Baxter NN: Stage-specific effect of adjuvant therapy following gastric cancer resection: A population-based analysis of 4,041 patients. Ann Surg Oncol 15: 500-507, 2008

16. Japanese Gastric Cancer Association: Japanese classification of gastric carcinoma-2nd english edition. Gastric Cancer 1: 10-24, 1998.

17. Sasako M, Sakuramoto S, Katai H, Kinoshita T, Furukawa H, Yamaguchi T, Nashimoto A, Fujii M, Nakajima T and Ohashi Y: Five-year outcomes of a randomized phase III trial comparing adjuvant chemotherapy with $\mathrm{S}-1$ versus surgery alone in stage II or III gastric cancer. J Clin Oncol 29: 4387-4393, 2011.

18. Noh SH, Park SR, Yang HK, Chung HC, Chung IJ, Kim SW, Kim HH, Choi JH, Kim HK, Yu W, et al: Adjuvant capecitabine plus oxaliplatin for gastric cancer after D2 gastrectomy (CLASSIC): 5-year follow-up of an open-label, randomised phase 3 trial. Lancet Oncol 15: 1389-1396, 2014.

19. GASTRIC (Global Advanced/Adjuvant Stomach Tumor Research International Collaboration) Group, Paoletti X, Oba K, Burzykowski T, Michiels S, Ohashi Y, Pignon JP, Rougier P, Sakamoto J, Sargent D, et al: Benefit of adjuvant chemotherapy for resectable gastric cancer: A meta-analysis. JAMA 303: 1729-1737, 2010.

20. Dudeja V, Habermann EB, Abraham A, Zhong W, Parsons HM, Tseng JF and Al-Refaie WB: Is there a role for surgery with adequate nodal evaluation alone in gastric adenocarcinoma? J Gastrointest Surg 16: 238-247, 2012.

21. Seyedin S, Wang PC, Zhang Q and Lee P: Benefit of adjuvant chemoradiotherapy for gastric adenocarcinoma: A SEER population analysis. Gastrointest Cancer Res 7: 82-90, 2014.

22. Datta J, McMillan MT, Ruffolo L, Lowenfeld L, Mamtani R, Plastaras JP, Dempsey DT, Karakousis GC, Drebin JA, Fraker DL and Roses RE: Multimodality therapy improves survival in resected early stage gastric cancer in the United States. Ann Surg Oncol 23: 2936-2945, 2016.

23. Wang Z, Yan J, Hu W, Zhang J and Huo B: Adjuvant chemotherapy provided survival benefit for stage T2N0 gastric cancer with high-risk factors. Neoplasma 65: 592-598, 2018.

24. Du C, Zhou Y, Huang K, Zhao G, Fu H and Shi Y: Defining a high-risk subgroup of pathological T2N0 gastric cancer by prognostic risk stratification for adjuvant therapy. J Gastrointest Surg 15: 2153-2158, 2011.

25. Park JH, Ryu MH, Kim HJ, Ryoo BY, Yoo C, Park I, Park YS, Oh ST, Yook JH, Kim BS and Kang YK: Risk factors for selection of patients at high risk of recurrence or death after complete surgical resection in stage I gastric cancer. Gastric Cancer 19: 226-233, 2016

26. Hwang JE, Hong JY, Kim JE, Shim HJ, Bae WK, Hwang EC, Jeong O, Park YK, Lee KH, Lee JH, et al: Prognostic significance of the concomitant existence of lymphovascular and perineural invasion in locally advanced gastric cancer patients who underwent curative gastrectomy and adjuvant chemotherapy. Jpn J Clin Oncol 45: 541-546, 2015.

27. Araki I, Hosoda K, Yamashita K, Katada N, Sakuramoto S, Moriya H, Mieno H, Ema A, Kikuchi S, Mikami T and Watanabe M: Prognostic impact of venous invasion in stage IB node-negative gastric cancer. Gastric Cancer 18: 297-305, 2015.

28. Yokoyama T, Kamada K, Tsurui Y, Kashizuka H, Okano E, Ogawa S, Obara S and Tatsumi M: Clinicopathological analysis for recurrence of stage Ib gastric cancer (according to the second english edition of the Japanese classification of gastric carcinoma). Gastric Cancer 14: 372-377, 2011.

29. Ohtsu A and Sasako M: Overview of adjuvant therapy for resected gastric cancer: Differences in Japan and the United States. Semin Oncol 32 (6 Suppl 9): S101-S104, 2005. 
30. Wang Y, Zhang J, Guo S, Dong Z, Meng X, Zheng G, Yang D, Zheng $Z$ and $Z$ hao $Y$ : Implication of lymph node staging in migration and different treatment strategies for stage T2NOMO and T1N1M0 resected gastric cancer: A SEER population analysis. Clin Transl Oncol 21: 1499-1509, 2019.

31. In H, Kantor O, Sharpe SM, Baker MS, Talamonti MS and Posner MC: Adjuvant therapy improves survival for T2N0 gastric cancer patients with sub-optimal lymphadenectomy. Ann Surg Oncol 23: 1956-1962, 2016.

32. Aoyama T, Yoshikawa T, Fujikawa H, Hayashi T, Ogata T, Cho H, Yamada T, Hasegawa S, Tsuchida K, Yukawa N, et al: Prognostic factors in stage IB gastric cancer. World J Gastroenterol 20: 6580-6585, 2014.

33. Sunakawa Y and Lenz HJ: Molecular classification of gastric adenocarcinoma: Translating new insights from the cancer genome atlas research network. Curr Treat Options Oncol 16: 17, 2015.

34. Polom K, Marano L, Marrelli D, De Luca R, Roviello G, Savelli V, Tan P and Roviello F: Meta-analysis of microsatellite instability in relation to clinicopathological characteristics and overall survival in gastric cancer. Br J Surg 105: 159-167, 2018.

35. Choi YY, Kim H, Shin SJ, Kim HY, Lee J, Yang HK, Kim WH, Kim YW, Kook MC, Park YK, et al: Microsatellite instability and programmed cell death-ligand 1 expression in stage II/III gastric cancer: Post hoc analysis of the CLASSIC randomized controlled study. Ann Surg 270: 309-316, 2019.

36. Miceli R, An J, Di Bartolomeo M, Morano F, Kim ST, Park SH, Choi MG, Lee JH, Raimondi A, Fucà G, et al: Prognostic impact of microsatellite instability in asian gastric cancer patients enrolled in the ARTIST trial. Oncology 97: 38-43, 2019.
37. Dai D, Zhao X, Li X, Shu Y, Shen B, Chen X, Chen D and Wang D: Association between the microsatellite instability status and the efficacy of postoperative adjuvant chemoradiotherapy in patients with gastric cancer. Front Oncol 9: 1452, 2020.

38. Lordick F: Chemotherapy for resectable microsatellite instability-high gastric cancer? Lancet Oncol 21: 203, 2020.

39. Van't Veer LJ, Dai H, van de Vijver MJ, He YD, Hart AA, Mao M, Peterse HL, van der Kooy K, Marton MJ, Witteveen AT, et al: Gene expression profiling predicts clinical outcome of breast cancer. Nature 415: 530-536, 2002.

40. Nakamura K, Hatakeyama K, Furukawa K, Fujiya K, Kamiya S, Hikage M, Tanizawa Y, Bando E, Ohshima K, Urakami K, et al: Prediction of S-1 adjuvant chemotherapy benefit in stage II/III gastric cancer treatment based on comprehensive gene expression analysis. Gastric Cancer 23: 648-658, 2020.

41. Cheong JH, Yang HK, Kim H, Kim WH, Kim YW, Kook MC, Park YK, Kim HH, Lee HS, Lee KH, et al: Predictive test for chemotherapy response in resectable gastric cancer: A multi-cohort, retrospective analysis. Lancet Oncol 19: 629-638, 2018.

42. Roskoski R Jr: The ErbB/HER family of protein-tyrosine kinases and cancer. Pharmacol Res 79: 34-74, 2014.

43. Begnami MD, Fukuda E, Fregnani JH, Nonogaki S Montagnini AL, da Costa WL Jr and Soares FA: Prognostic implications of altered human epidermal growth factor receptors (HERs) in gastric carcinomas: HER2 and HER3 are predictors of poor outcome. J Clin Oncol 29: 3030-3036, 2011.

44. Wang S, Zheng G, Chen L and Xiong B: Effect of HER-2/neu over-expression on prognosis in gastric cancer: A meta-analysis. Asian Pac J Cancer Prev 12: 1417-1423, 2011. 